\title{
EVALUACIÓN DE LA PRODUCCIÓN DE LÁTEX DE SANGRE DE GRADO (Croton lechleri) EN FUNCIÓN AL DIÁMETRO Y CUATRO PERIODOS DE PRECIPITACIÓN EN POBLACIONES NATURALES DE UCAYALI, PERÚ
}

\author{
EVALUATION OF THE “DRAGON'S BLOOD” (Croton lechleri) LATEX \\ PRODUCTION, TAKING INTO CONSIDERATION ITS DIAMETER AND FOUR \\ PERIODS OF RAINFALL IN NATURAL POPULATIONS FROM UCAYALI, PERÚ
}

\author{
Andrés Castillo-Quiliano y Gilberto Domínguez-Torrejón ${ }^{2}$
}

\begin{abstract}
Resumen
El látex de sangre de grado (Croton lechleri), que se extrae y comercializa actualmente en el Perú, proviene de poblaciones naturales existentes en bosques secundarios de la Amazonía. La producción por árbol ha sido poco estudiada, a pesar de que esta información es indispensable para determinar los turnos de cosecha y la rentabilidad de las plantaciones.

El presente trabajo tuvo como objetivo determinar el volumen de producción de látex. Se aplicó el método del tumbado y sangrado, tomando en cuenta rangos de diámetro a la altura del pecho (DAP): $15-20,21-25,26-30$ y >30 cm, y cuatro periodos de extracción del látex determinado por los niveles de precipitación: fines de lluvia (mayo), periodo seco (septiembre) inicio de lluvias (diciembre) y plena lluvia (marzo).

El estudio se realizó con la participación de agricultores en bosques secundarios de las regiones de Ucayali y Mariscal Cáceres. Se determinó que el volumen de producción de látex es directamente proporcional al diámetro y a la variación de los niveles de precipitación. Se encontró también la existencia de una alta variabilidad en la producción de látex por árbol, dentro y entre los cuatro rangos dimétricos. Adicionalmente se observó la presencia de tres variedades de látex diferenciados por propiedades organolépticas como color (rojo sangre, vino-morado y ocre) y la densidad de los mismos.

Se recomienda realizar extracciones de látex en el periodo diciembre a mayo, que son los meses de mayor producción así como realizar estudios fitoquímicos relacionados con la presencia de principios activos.
\end{abstract}

Palabras clave: Sangre grado, Croton, Croton lechleri, extracción de látex, producción de látex, rendimiento de látex.

\begin{abstract}
Dragon's blood latex (Croton lechleri Muell.Arg), is extracted and commercialized in Perú and it largely comes from natural populations in the Amazon's secondary forests. Each tree latex production has been scarcely studied, in spite of the fact that this information is essential for determining crop shifts and profitability of plantations.

This study was aimed at determining the volume of latex production. The knocked over and bleeding method was applied, taking into account the following diameter ranges at the height of the chest (DAP): 15-20, 21-25, 26-30 and > $30 \mathrm{~cm}$; and at four latex extraction periods determined by rainfall levels: end of rainfall (May), dry period (September), beginning of rainfall (December) and middle of rainfall (March).

The study was conducted with the participation of secondary forest farmer from Ucayali and Mariscal Cáceres regions. It was found out that the volume of latex production is directly proportional to the diameter of the tree and to the variation of the rainfall levels. The existence of a high variability in the latex production per tree, among and within each the four diameter ranges was found. Additionally the presence of three varieties of latex differentiated by their organoleptic characteristics such as color (red blood, wine-purple color and ochre) and density were observed.

Latex harvesting from December to May is recommended, because they are the months of highest production. Phytochemical studies related to the presence of active ingredients are also recommended.
\end{abstract}

Key words: Dragon blood, Croton, Croton lechleri, latex extraction, latex production, latex performance. 


\section{Introducción.}

Sangre de grado (Croton lechleri), es una especie forestal amazónica que en los últimos años ha incrementado su demanda en el mercado nacional e internacional por las propiedades medicinales atribuidas al látex en la cicatrización de heridas y en el tratamiento de afecciones estomacales como ulceras.

La producción actual del látex, procede de árboles de regeneración natural existentes en los bosques secundarios de la Amazonía y, escasamente de árboles plantados. Por su creciente importancia económica, es una alternativa interesante para los agricultores producir este látex medicinal mediante su instalación en sistemas de producción agroforestal y manejo en los bosques secundarios de los agropecuarios; para ello es importante conocer las características de la producción de látex, que van a definir el desarrollo de paquetes tecnológicos que demuestren rentabilidad y sostenibilidad de la especie (Nalvarte et al., 1999).

Es de suponer que la mayor producción de látex está en relación directa al diámetro del árbol; sin embargo, es también cierto que a mayor diámetro, mayor tiempo de espera para la cosecha y recuperación de la inversión. Es decir que para lograr árboles con diámetros mayores a 40 ó $50 \mathrm{~cm}$, se requieren de periodos mayores a 20 años; de esta manera, muchos agricultores no se interesan en manejar la especie en tanto no se haya determinado el turno óptimo para su cosecha y el rendimiento en volumen de látex por árbol.

La "Sangre de grado" está distribuida en toda América tropical y sub-tropical, desde la amazonía Peruana hasta las Guyanas. En América del Sur se le encuentra en Bolivia, Colombia, Ecuador y Perú (Pinedo et al, 1997; Meza, 1999). En el Perú se encuentra en los departamentos de Loreto en las cuencas del río Amazonas (Llachapa, río Napo; Indiana, Padre cocha, Morona, río Nanay) y diferentes localidades de San Martín, Huánuco, Cerro de Pasco (Oxapampa, Satipo, Puerto Bermúdez, Iscozacin, Villarica), Junín (Chanchamayo), Cuzco, Puno y Ucayali (Pucallpa).

Existe en el Perú al menos cinco especies con propiedades de producir látex medicinal: Croton lechleri, C. draconoides, C. erytrochilus, C. palanostigma, C. huitotorum, Siendo las características dendrológicas muy similares entre ellas (Meza, 1999). En condiciones naturales de bosques secundarios se le encuentra asociada con más frecuencia a: cetico (Cecropia spp.), bolaina blanca (Guazuma crinita), capirona (Calycophyllum spruceanum), shimbillos (Inga spp.), oje (Ficus insipida), topa Ochroma piramidale, aucatadijo (Trema micranta), Llausa quiro (Heliocarpus spp.). Así mismo asociado se la encontró con cultivos como el café Coffea arabiga, el plátano y también con pasto Braquiaria spp. (Ravelo, 1992).
La "sangre de grado o drago", como se conoce en toda América Latina, es un árbol que produce un látex rojo que tiene componentes químicos que actúan en el tratamiento de varias enfermedades, tales como, diarreas, úlceras y en la cicatrización de heridas. Los principios activos del látex incluyen a la taspina y alcaloides antitumorales como: piridone, aportine indole, quinolin, tropanes, ácido graso insaturado, antraquinonas y triterpenos. El látex también contiene compuestos fenólicos con fuertes cualidades antisépticas. La "sangre de grado" es comúnmente mezclada con agua y tomada internamente como un tratamiento para úlceras del estómago (Chen $\mathrm{Zp}$, 1994).

Respecto a su regeneración natural, Ravelo (1994) reporta de 16 a 72 individuos por hectárea, con DAP promedios de $26.3 \mathrm{~cm}$ por parcela; mientras que Gaviria (1995) reporta poblaciones naturales en diferentes partes del Perú con densidades de 5 arb./ha en el Palcazu, 88.57 arb./ha en Oxapampa, 149 arb./ha en áreas sin intervención humana de Madre de Dios, 42 arb./ha con ligera intervención y 57arb./ha con sustancial intervención.

De otro lado, Ríos (2006), reporta de 10 a 33 árboles por hectárea en suelos Entisoles y inceptisoles del bosque Nacional Alexander von Humbold. (Phillips 1991 citado por Ríos 2006), indica que Croton nunca crece lejos de un curso permanente de agua.

Según Nalvarte et al. (1999), el rendimiento está en función del tamaño del árbol, a mayor diámetro, mayor rendimiento. Meza (1998) señala que la cosecha puede ejecutarse a partir del séptimo u octavo año de plantado, cuando el árbol mide aproximadamente $30 \mathrm{~cm}$ de DAP.

El método tradicional de extracción del látex con fines comerciales es, tumbar el árbol y "sangrar" el fuste totalmente; esta operación implica hacer cortes en anillos en el fuste, lo cual permite que el látex fluya libremente por gravedad. El rendimiento está en función del tamaño del árbol, a mayor diámetro, mayor rendimiento, considerando un diámetro mínimo de aprovechamiento de $20 \mathrm{~cm}$ (Ravelo, 1992). Gaviria (1995), menciona que este DAP se alcanza a los 10 años de edad pero que la producción de cada árbol es difícil de estimar. Por ejemplo, en selva central durante la época lluviosa en una zona inundable y en una jornada de mañana, un árbol de $20 \mathrm{~cm}$ puede proporcionar $65 \mathrm{ml}$ de látex, uno de $35 \mathrm{~cm}, 250 \mathrm{ml} \mathrm{y}$ de $50 \mathrm{~cm}$ se puede obtener hasta dos litros. Sin embargo, en el Valle del Huallaga (zona de Tocache), que es un lugar muy lluvioso, se obtiene 2 litros por árbol de 5 años de edad, lo cual es muy difícil que a esta edad alcance por lo menos los $35 \mathrm{~cm}$ de DAP (Mendivel, 1998, citados por Nalvarte et al., 1999).

Las horas recomendables para colectar es a partir de las 5 a 7 am haciendo cortes anillados distanciados 
$15 \mathrm{~cm}$ en el fuste. El látex no debe almacenarse por más de 2 meses porque afecta su calidad (Meza, 1998)

Los niveles de producción son muy variables. Forero (1992), realizó un estudio en Colombia sobre la relación del DAP y la productividad, donde encontró los siguientes resultados:

\begin{tabular}{l}
\multicolumn{5}{c}{$\begin{array}{l}\text { Volumen de producción de látex en } \\
\text { relación al diámetro (Dap) }\end{array}$} \\
\begin{tabular}{cccc}
\hline $\mathrm{N}^{\circ}$ & $\begin{array}{c}\text { DAP } \\
\text { (cm) }\end{array}$ & $\begin{array}{c}\text { Vol. Látex } \\
\text { (cm3) }\end{array}$ & $\begin{array}{c}\text { Corteza seca } \\
\text { (g) }\end{array}$ \\
\hline 01 & 20 & 155 & 470 \\
02 & 23 & 135 & -- \\
03 & 25 & 130 & 500 \\
04 & 25 & 140 & 560 \\
05 & 25 & 180 & 460 \\
06 & 35 & 190 & 510 \\
07 & 40 & 210 & 710 \\
\hline
\end{tabular}
\end{tabular}

En Ecuador, Ravelo (1992) reporta a partir de una encuesta, que el $7 \%$ de encuestados señala que de un árbol de 20 - $30 \mathrm{~cm}$ de DAP se extrae un litro de látex, el $44 \%$ dice que de árboles de $30-40 \mathrm{~cm}$ se extrae 1,7 a 2 litros y el $33 \%$ que de árboles grandes con DAP de 40 - $50 \mathrm{~cm}$ se extraen 5,0 a 5,1 litros. Con respecto al método de cosecha, el mismo autor señala tres métodos: 1) Tumbando el árbol y sangrado total, recogiéndose el látex sobre plástico. 2) Mediante incisiones a la corteza de árboles en pie; no es muy común cuando se cosecha para el comercio, pero señala que sería el más recomendable, dado que permite tener los árboles por mayor tiempo en producción; es común en las poblaciones Quichuas del Ecuador. 3) Uso de clavos con los cuales pican la corteza, para extraer para autoconsumo y comercializar en pocas cantidades.

Refiriéndose al segundo método, Atanasio y Domínguez (2000), en un estudio realizado en tres lugares ubicados a 285, 362 y 1250 msnm y con diferentes tipos de suelo, muestran diferencias en la producción de látex, las cuales se incrementan en función del diámetro. La técnica de extracción aplicado fue de incisiones en " $V$ " con dos técnicas de exudación (natural y aplicando el estimulante ethrel), realizadas a razón de 5 incisiones interdiarias/árbol /año. En un total de 36 árboles, se alcanzó rendimientos promedio de $50.58 \mathrm{ml} /$ árbol sin aplicar

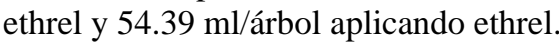

King et al. (1997), al comparar el rendimiento del látex de Croton lechleri entre los métodos de "tumbado del árbol" y el de corte en "V" (shiringuero), con árboles de $55 \mathrm{~cm}$ de DAP y corte a la altura del pecho, obtuvieron 4 litros de látex por árbol en el primer método y en el segundo menos de 1 litro.

Se observa la diferencia entre ambos, solo que con el segundo método no se debe de esperar alcanzar los $55 \mathrm{~cm}$ de DAP para iniciar la cosecha y ésta no sería una sola cosecha, mientras que con el primero la extracción es destructiva.

Del mismo modo, Gudiño et al. (1991), en su estudio sobre el manejo de Croton spp., en Ecuador, encontró $12 \%$ de rendimiento con el método shiringuero y $80 \%$ tumbando el árbol.

Pinedo et al. (1997), manifiestan que los factores que mayormente influyen en el rendimiento del látex de Croton lechleri son: radiación solar, diámetro del árbol, follaje, ángulo de corte, precipitación y fase lunar, siendo lo mas conveniente entre cuarto creciente y luna llena. Así mismo, considera que una plantación alcanza rendimientos económicamente rentables a partir del octavo año de plantación.

El objetivo del estudio fue determinar la producción de látex de sangre de grado en relación al diámetro a la altura del pecho (DAP) mediante la técnica del tumbado y el sangrado total, a través de cortes en anillos en todo el fuste, realizada en cuatro periodos estacionales determinados por la abundancia de precipitación.

Los resultados permitirán dilucidar y recomendar los diámetros y los meses más apropiados para cosechar el látex de Sangre de grado en poblaciones naturales de bosques secundarios.

\section{Materiales y métodos. \\ Localización.}

La investigación fue realizada en la Provincia de Padre Abad y Puerto Inca, Distritos de Irazola y Puerto Inca; de las Regiones de Ucayali y Mariscal Cáceres respectivamente. El área de influencia corresponde al Bosque Nacional Alexander von Humboldt, se ubica entre 240 y $340 \mathrm{msnm}$, de la selva baja tropical. La temperatura media máxima es de $31{ }^{\circ} \mathrm{C}$, mínima de $21{ }^{\circ} \mathrm{C}$, media de $26{ }^{\circ} \mathrm{C}$. Humedad relativa media de $75 \%$ y las precipitaciones anuales superan a los $4000 \mathrm{~mm}$ (INIAA-JICA, 1991).

Los suelos predominantes en el área de trabajo son los Entisoles y Inceptisoles, de textura Franco Arcillo limoso y Arcillo limoso, de moderadamente a bien drenado. La mayor abundancia de árboles/ha de sangre de grado, se da donde el $\mathrm{pH}$ esta entre 4,10 y 4,50 con 30 y 33 árboles respectivamente (Ríos, 2006).

Los árboles para el estudio fueron ubicados en bosques secundarios de 6 a 12 años de edad, según la información proporcionada por los dueños de estas parcelas, quienes junto a otros miembros de su familia tuvieron participación directa en los trabajos de campo. Los Caseríos donde se ubicaron los bosques secundarios se distribuyen a lo largo de la Carretera Federico Basadre (CFB), como se describe en la Tabla 1.

Metodología.

Se aplicó el método descriptivo, para determinar las características dasométricas de los árboles, clasificándolos por clases diamétricas; y el método 
Tabla 1: Descripción de las localidades seleccionadas para el estudio.

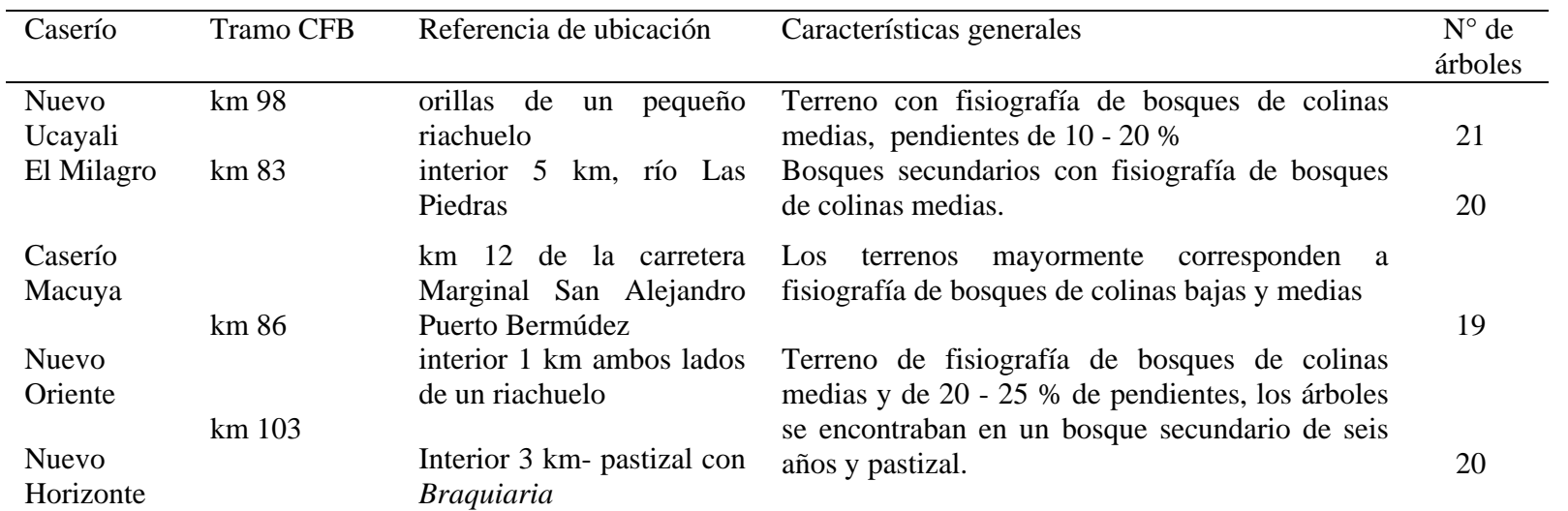

analítico, en relación a la producción del látex de cuatro clases diamétricas: 15-20, 21-25, 26-30 y >30 $\mathrm{cm}$, y en cuatro periodos de precipitación: fines de lluvia (mayo), periodo seco (septiembre), inicio de lluvia (diciembre) y plena lluvia (marzo). La técnica de extracción de látex fue el tumbado del árbol y sangrado total realizando cortes circulares en el tronco, recogiéndose el látex en lámina de plástico colocando debajo del fuste. Las horas de extracción fueron de 6 am a $12 \mathrm{~m}$.

Población, tamaño de muestra y variables evaluadas

1. Población. Las poblaciones evaluadas fueron árboles de sangre de grado presentes en bosques secundarios, que según Ríos (2006) fluctúa entre 10 a 33 árboles/ha, ubicados en predios de agricultores y donde la presencia en forma natural de esta especie es frecuente, en algunos casos muy homogéneos y de edades que fluctuaron, entre 6 -12 años.

2. Tamaño de muestra. El tamaño de la muestra considerada a ser evaluada por cada periodo de aprovechamiento fue de veinte (20) árboles, distribuidos en cinco (5) árboles por cada clase diamétrica siguiente: de 15 -20, 21-25, 26-30 y $>30 \mathrm{~cm}$.

Se extrajo el látex de ochenta (80) árboles en total, distribuido de la siguiente manera:

- 21 árboles fines de lluvia (mayo),

- 20 árboles periodo seco (septiembre),

- 19 árboles inicio de lluvia (diciembre) y

- 20 árboles plena lluvia (marzo).

3. Variables evaluadas. Las variables que fueron evaluadas en el estudio fueron: Diámetro a la altura del pecho (DAP) en centímetros, altura total y altura comercial en metros después del tumbado del árbol, la producción y color del látex por árbol, tiempo de aprovechamiento según clase de diámetro, grosor de corteza en milímetros medido con vernier.

4. Procedimiento. Se contactó y coordinó con cada uno de los cinco agricultores para realizar el estudio, se buscó la participación del grupo familiar para ubicar áreas con poblaciones de árboles en las diferentes categorías dentro de su predio. Ubicados los árboles se numeró en forma correlativa con números romanos a la clase diamétrica que le correspondía y con números arábigos al árbol de la clase diamétrica.

Previo al tumbado de cada árbol, se eliminaron las lianas y vegetación circundante, el tumbado se realizó con hacha cortando lo más bajo posible, 15 - $20 \mathrm{~cm}$. del suelo; despuntando la copa hasta donde sea posible de aprovechar para extraer el látex e inclusive ramas gruesas (árboles ramificados desde muy abajo), limpieza de la maleza, de tal manera que el fuste quede libre y limpio. La extracción del látex se realizó practicando cortes con el machete y apoyado con un mazo de madera dura, distanciados de 10 - 15 cm en forma circular y perpendicular al eje del fuste, según la recomendación de Meza (1998). Para recoger el látex se utilizo láminas de plástico confeccionado en forma de camilla y extendiéndose debajo del fuste a extraerse.

\section{Resultados y discusión.}

Características del látex:

Las características dendrológicas de hojas y corteza de los árboles de "sangre de grado" aprovechados, son muy similares. Siendo la misma especie, no se esperaría diferencias en otras características dendrológicas; sin embargo se encontraron diferencias en la coloración del látex (rojo sangre, vino-morado y ocre), densidad (más denso y menos denso) y facilidad de secreción después del corte de extracción. No se puede señalar con precisión si las tres variedades de látex observados corresponden a diferentes especies, a variedades de una sola especie, ó posiblemente eco tipos. Se ha registrado algunas diferencias en las hojas en las diferentes muestras colectadas, estas fueron enviadas al herbario Museo Paraense Emilio Goeldi -Belem Brasil y los resultados alcanzados por el Dr. Ricardo Secco muestran que todas corresponden a Croton lechleri. 
Las tres variedades de látex fueron encontradas en las diferentes localidades y periodos de aprovechamiento: El color rojo sangre es el más representativo y apreciado por los compradores, por sus características organolépticas y facilidad de extracción, fluye fácilmente después del corte, aunque en algunos casos es tan denso que se adhiere al plástico donde es recogido y fácilmente cristaliza con la luz solar, al mezclarse con el agua se torna color rojo claro. El de color vino o morado, fluye al corte con formaciones de espuma blanquecina al inicio, con tendencia a coagularse, resbala fácilmente en el plástico y cuando es envasado en el frasco es de color vino (morado oscuro). El color ocre, es muy difícil de fluir después del corte porque coagula rápidamente en el fuste y en el plástico, en el frasco es de color ladrillo, por estas características y el color mismo no es apreciado por los compradores porque suponen que está mezclado con agua.

De acuerdo a la muestra evaluada se determinó una proporción de frecuencia de 3:1:1 hasta la tercera extracción, sin embargo en la última cosecha (Caserío Macuya), la variedad morada fue el más frecuente $(2: 7: 1)$, esto puede ser un indicador de efectos micro diferencia está entre la clase diamétrica de 15 - 20 y 21-25 cm (2.30 m); y menor entre la clase 21- $25 \mathrm{~cm} \mathrm{y}$ las dos últimas $(0.70$ y $0.75 \mathrm{~m})$ (Tabla 2). Sin embargo, se debe señalar que las condiciones de crecimiento de los árboles aprovechados en el tercer sitio fueron a campo abierto, en pastizales de Braquiaria spp. (9 árboles), donde la característica más saltante fueron fustes comerciales de baja altura y presentaban ramificaciones bajas y gruesas, las cuales también fueron aprovechados, pero demandó más trabajo.

En el caso de los otros lugares los árboles de Croton lechleri se encontraban en bosques secundarios, siendo los fustes de mayores alturas comerciales, largos y rectos sin ramificaciones bajas y asociados con especies típicas de este tipo de bosque. Grosor de corteza:

Esta variable es muy importante, dado que aquí se encuentra el producto de interés por aprovecharse; la tendencia encontrada en promedio es a incrementarse de 4 a $6 \mathrm{~mm}$, a medida que el diámetro aumenta, siendo directamente proporcional al diámetro del árbol (Tabla 2).

Tabla 2. Variables medidas en el aprovechamiento del látex de ochenta árboles de sangre de grado.

\begin{tabular}{ccccccc}
\hline \multirow{2}{*}{$\begin{array}{c}\text { Clase } \\
\text { diamétrica } \\
(\mathrm{cm})\end{array}$} & \multicolumn{5}{c}{ Promedios por clase diamétrica } \\
\cline { 2 - 7 } & Árboles & $\begin{array}{c}\text { Dap } \\
(\mathrm{cm})\end{array}$ & $\begin{array}{c}\text { Grosor de corteza } \\
(\mathrm{mm})\end{array}$ & $\begin{array}{c}\text { Altura Total } \\
(\mathrm{m})\end{array}$ & $\begin{array}{c}\text { Altura comercial } \\
(\mathrm{m})\end{array}$ & $\begin{array}{c}\text { Tiempo de } \\
\text { Extracción. }(\mathrm{min})\end{array}$ \\
\hline $15-20$ & 22 & 17.85 & 4.00 & 14.60 & 11.32 & $30-40$ \\
$21-25$ & 24 & 23.57 & 5.24 & 16.90 & 11.50 & $30-60$ \\
$26-30$ & 16 & 28.06 & 5.40 & 17.80 & 12.90 & $40-80$ \\
$>30$ & 18 & 33.62 & 6.10 & 17.65 & 12.65 & Hasta 120 \\
\hline
\end{tabular}

ambientales o variedades diferentes que debe ser corroborado con estudios posteriores.

Frecuencia de clases diamétricas encontradas:

La mayor frecuencia de árboles correspondieron a las categorías diamétricas de 15-20 y 21-25 cm con 22 y 24 árboles y en menor número en la clase 26-30 y > de $30 \mathrm{~cm}$. con 16 a 18 árboles respectivamente. No se encontraron árboles con diámetros mayores de $42 \mathrm{~cm}$ (Tabla 2). Esto se explica porque los sitios muestreados, son bosques secundarios en sus primeros estadios (6 - 12 años) y los agricultores no permiten barbechos de muchos años por la necesidad de uso del suelo para fines agrícolas, viéndose obligados a tumbarlos y en mucho de los casos sin aprovechar el látex o cuando todavía no alcanzaron diámetros de aprovechamiento. No se pudo delimitar tamaño de parcela porque son árboles de regeneración natural y se estima que en una hectárea se puede encontrar de 10 a 33 árboles /ha.

\section{Características de alturas totales y comerciales:}

La diferencia de las alturas totales y comerciales dentro de cada clase de diámetro es mínima, pero entre clases diamétricas si hay diferencias; tiende a ser mayor cuando se incrementa el diámetro. La mayor

\section{Variabilidad de producción:}

La desviación estándar calculada, determinó una alta variabilidad en la producción del látex por árbol dentro de cada clase de diámetro; así mismo entre las cuatro clases de diámetro la tendencia es a incrementarse cuando se incrementa el diámetro. Con respecto a los cuatro periodos de precipitación, en el periodo lluvioso la producción de látex es menos variable en comparación al periodo seco; así mismo se incrementa hacia los periodos de mayor precipitación. (Tablas 3, 4, 5 y 6).

Esta alta variabilidad registrada dentro de cada clase de diámetro, se puede atribuir a dos factores: la variabilidad genética de la especie y las condiciones de micro hábitat. El grado de cruzamiento al estado natural y el conocimiento de los progenitores es difícil de establecer dado que son bosques naturales. En el caso de las condiciones edafo-climáticas, por ser similares, el efecto en el desarrollo de los árboles en donde se realizó el aprovechamiento de látex puede no ser significativo (Ríos, 2006), sin embargo debe de considerarse más adelante posibles efectos micro ambientales relacionados con la características de los suelos y la capacidad de la planta de absorber 
nutrientes favorecidos por condiciones micro climáticas. Esta variabilidad representa ventajas para la selección masal, permitiendo seleccionar individuos con características deseables para la domesticación y programas de reforestación.

Tiempo de extracción de látex:

El tiempo de tumbado para la extracción de látex en el rango de diámetro de 15 - $20 \mathrm{~cm}$ es muy rápido. Un agricultor demora de 5 - 8 minutos para el tumbado, incrementándose el tiempo a medida que aumenta el diámetro; así tenemos que para árboles mayores a $30 \mathrm{~cm}$ se demora de 15 - 20 minutos. El trabajo de despuntado y limpieza del fuste de las malezas demora hasta 25 minutos, pero cuando esta libre como en los pastizales es únicamente despuntar la copa del árbol. El tiempo propiamente dicho de extracción es también proporcional al diámetro, en árboles de $15-20 \mathrm{~cm}$ es de 30 - 40 minutos y en árboles mayores $30 \mathrm{~cm}$ de diámetro puede demorar más de dos horas, dependiendo además de las condiciones donde y como cae el árbol; presencia de malezas o de otros árboles que dificultan la caída, posición y lugar de caída del árbol. El tiempo de secreción del látex después del corte no toma más de 20 minutos y lo que demora más es acomodar el fuste en una posición adecuada para extraer el látex; en los árboles de diámetro mayores a $30 \mathrm{~cm}$ el peso es una dificultad para acomodarlo en la posición adecuada. Los árboles de $25 \mathrm{~cm}$ de diámetro tienen más ventajas porque pueden ser manipulados fácilmente por dos personas para la extracción.

Las distancias de corte de secreción más recomendable fueron de 10 cm Para recolectar el látex resultó más apropiado el uso del plástico en forma de camilla, de $2.10 \mathrm{~m}$ de largo, $0.65 \mathrm{~m}$ de ancho y sujetados por los lados a dos varillas de $2.50 \mathrm{~m}$ de largo. Las horas de trabajo recomendable es de 6 am a $12 \mathrm{~m}$, con tres personas se extraen 5-6 árboles en este tiempo.

Producción de látex según clase diamétrica y periodo de precipitación:

El primer aprovechamiento fue realizado en el caserío Nuevo Ucayali a fines del periodo de lluvia (mayo). Se extrajo el látex de 21 árboles, con edades de siete años según información del dueño de la parcela y distribuidos de la siguiente manera: seis árboles en las categorías de 15 - 20 y $21-25$ cm, siete en la categoría de 26 - 30 cm, y 2 árboles mayores a $30 \mathrm{~cm}$ de DAP respectivamente.

En la Tabla 3, se puede observar la producción de látex por árbol y según la clase diamétrica: por ejemplo en la categoría diamétrica $15-20 \mathrm{~cm}$, la máxima producción por árbol es 300 cc y el mínimo 50 cc, en la de 26-30 la máxima es 1150 cc y 400 cc la mínima. La producción de látex entonces es directamente proporcional al diámetro. La variabilidad de la producción de látex entre árboles de la misma categoría diamétrica es alta, según la desviación estándar, de igual manera ocurre entre diferentes categorías. La producción total fue de 9.77 litros de látex.

La segunda extracción se realizó en el caserío El en el periodo de fines de lluvia (mayo).

\begin{tabular}{lcccccc}
\hline & \multicolumn{3}{c}{$\mathrm{N}^{\circ}$ Árboles/Clase diamétrica (cm) } & \multicolumn{2}{c}{ Totales } \\
\cline { 2 - 7 } & $15-20(\mathrm{I})$ & $21-25(\mathrm{II})$ & $26-30(\mathrm{III})$ & $>30(\mathrm{IV})$ & Arb./ clase & Latex (cc) \\
\cline { 2 - 7 } & 150 & 200 & 400 & 1225 & 4 & 1975 \\
& 250 & 345 & 900 & 1000 & 4 & 2495 \\
& 125 & 250 & 1150 & & 3 & 1525 \\
& 300 & 350 & 575 & & 3 & 1225 \\
& 150 & 325 & 800 & & 3 & 1275 \\
& 50 & 275 & 475 & & 3 & 800 \\
\hline S & 1025 & 1745 & 4775 & 2225 & 21 & 9770 \\
Prom. & $\mathbf{1 7 1 . 0 0}$ & $\mathbf{2 9 1}$ & $\mathbf{6 8 2}$ & $\mathbf{1 1 1 2 . 5 0}$ & & $\mathbf{4 6 5 . 2 4}$ \\
D. S & 90.02 & 57.62 & 276.03 & & & \\
\hline
\end{tabular}

Milagro, correspondiente al periodo climático seco (septiembre). Se aprovecharon 20 árboles, distribuidos de la siguiente manera: seis árboles en las categorías de $15-20$ y $21-25 \mathrm{~cm}$, dos árboles en $26-30 \mathrm{~cm}$ y seis árboles mayores a $30 \mathrm{~cm}$ de DAP (Tabla 4).

En los resultados se vuelve a observar la alta

Tabla 4. Producción (cc) de látex de sangre de grado por árbol y clase de diámetro en el periodo seco (septiembre)

\begin{tabular}{lcccccc} 
& \multicolumn{3}{c}{$\mathrm{N}^{\circ}$ Árboles/Clase diámetrica (cm) } & \multicolumn{2}{c}{ Totales } \\
\cline { 2 - 7 } & $15-20(\mathrm{I})$ & $21-25(\mathrm{II})$ & $26-30(\mathrm{III})$ & $>30(\mathrm{IV})$ & Arb./clase & Latex (cc) \\
\cline { 2 - 7 } & 40 & 175 & 275 & 525 & 4 & 1015 \\
& 100 & 10 & 520 & 150 & 4 & 780 \\
& 125 & 200 & - & 550 & 3 & 875 \\
& 100 & 125 & - & 250 & 3 & 475 \\
& 10 & 150 & - & 675 & 3 & 835 \\
& 80 & 250 & - & 300 & 3 & 630 \\
\hline S & 455 & 910 & 795 & 2450 & 20 & 4610 \\
Prom. & $\mathbf{7 5 . 8 3}$ & $\mathbf{1 5 1 . 6 7}$ & $\mathbf{3 9 7 . 5 0}$ & $\mathbf{4 0 8 . 3 3}$ & & $\mathbf{2 3 0 . 5 0}$ \\
DS & 42.94 & 81.65 & 173.24 & 204.12 & & \\
\hline
\end{tabular}

variabilidad de la producción entre árboles dentro de una misma clase diamétrica, así como entre clases de diámetro; la producción total de látex fue de 4.61 litros, con una disminución del 53\% en comparación 
al periodo del mes de mayo que fue de 9.77 litros, lo que nos indica que la producción es fuertemente afectada por el periodo de sequía, propio de los meses de julio, agosto y septiembre en la región de Ucayali.

La tercera extracción de látex correspondió al periodo de inicio de lluvia (diciembre) y se realizó en los caseríos de Nuevo Oriente y Nuevo Horizonte. Se aprovecharon árboles, $\quad 10 \quad 19$ encontraban en un bosque secundario de 7 años de edad y 9 en pastizales de Braquiaria spp., cuya edad según su propietario tenía 12 años y mayormente correspondieron a la categoría mayor de $30 \mathrm{~cm}$ de DAP.

Del total de árboles aprovechados 5 son de la clase diamétrica 15-20 cm, 6 de la clase de $21-25 \mathrm{~cm}, 3$ de la clase $26-30 \mathrm{~cm}$ y 5 mayores a $30 \mathrm{~cm}$ de DAP. Respecto a la producción de látex, las tendencias de la alta variabilidad de producción por árbol dentro y entre las clases diamétricas se vuelven a repetir. (Tabla 5).

El total de látex extraído en el periodo alcanzó a 8.44 litros, observándose un incremento del $83 \%$ en relación al periodo seco.

La cuarta extracción correspondió al periodo climático de plena lluvia (marzo) y se ejecutó en el Caserío Macuya. Se aprovechó 20 árboles distribuidos en las siguientes categorías diamétricas: cinco árboles en $15-20 \mathrm{~cm}$ y mayor a 30 $\mathrm{cm}$, seis árboles en 21-25 cm y cuatro árboles en la categoría 26-30 cm. La producción total de látex extracción. extraído en el periodo alcanzo a 9.32 litros, incrementándose ligeramente en 10 \% en comparación al periodo de diciembre (Tabla 6). en el periodo de plena lluvias (marzo).

\begin{tabular}{lcccccc}
\hline & \multicolumn{3}{c}{$\mathrm{N}^{\circ}$ Arboles/Clase de diámetro (cm) } & \multicolumn{2}{c}{ Totales } \\
\cline { 2 - 7 } & $15-20(\mathrm{I})$ & $21-25(\mathrm{II})$ & $26-30(\mathrm{III})$ & $>30(\mathrm{IV})$ & Arb./clase & Látex (cc) \\
\cline { 2 - 7 } & 150 & 475 & 325 & 875 & 4 & 1825 \\
& 150 & 200 & 975 & 1250 & 4 & 2575 \\
& 58 & 475 & 450 & 775 & 4 & 1758 \\
& 170 & 450 & 450 & 1050 & 4 & 2120 \\
& 50 & 375 & & 375 & 3 & 800 \\
& 578 & 240 & & & 1 & 240 \\
\hline S & 5215 & 2200 & 4325 & 20 & 465.9 \\
Prom. & 115.6 & 369 & 550 & 865 & & \\
DS & 56.87 & 121.90 & 280.16 & 328.16 & & \\
\hline
\end{tabular}

Producción de látex por clase diamétrica en los cuatro periodos de extracción.

Los rangos de producción registrados en cada clase de diámetro se puede ver en el Tabla 7; hay clases diamétricas en donde la variabilidad entre árboles pueden diferenciarse entre seis o doce veces del volumen de látex cosechado en función del periodo de extracción, como se puede notar en la categoría de 15 - $20 \mathrm{~cm}$, periodo fines de lluvia, y época seca, respectivamente. En la categoría 21-25 cm época seca, la diferencia entre la producción mínima y máxima es aún mayor, 25 veces (10 a 250 cc), disminuyendo esta tendencia a medida que aumenta la clase diamétrica.

Teniendo en cuenta el total de litros cosechados se puede decir que el inicio de la producción es la época

Tabla 7. Rango de producción (cc) de látex por clase de DAP y periodos de

\begin{tabular}{ccccc}
\hline \multirow{2}{*}{$\begin{array}{c}\text { Clase diamétrica } \\
(\mathrm{cm} .)\end{array}$} & \multicolumn{4}{c}{ Rango de producción (cc) de látex/periodo de extracción. } \\
\cline { 2 - 5 } & $\begin{array}{c}\text { Fines de lluvias } \\
\text { (mayo) }\end{array}$ & $\begin{array}{c}\text { Época seca } \\
\text { (septiembre) }\end{array}$ & $\begin{array}{c}\text { Inicio de lluvias } \\
\text { (diciembre) }\end{array}$ & $\begin{array}{c}\text { Plena lluvia } \\
\text { (marzo) }\end{array}$ \\
\hline $15-20$ & $50-300$ & $10-125$ & $50-200$ & $50-170$ \\
$21-25$ & $200-350$ & $10-250$ & $100-375$ & $200-475$ \\
$26-30$ & $400-1150$ & $275-520$ & $450-750$ & $325-975$ \\
$>30$ & $1000-1225$ & $150-675$ & $450-1550$ & $375-1250$ \\
\hline Total (litros) & 9,77 & 4,61 & 8,44 & 9,32 \\
& $-53 \%$ & $83 \%$ & $10.5 \%$ & $4.8 \%$ \\
\hline
\end{tabular}

Tabla 5. Producción (cc) de látex de sangre de grado por árbol y clase de diámetro a Inicio de lluvias (Diciembre).

\begin{tabular}{lcccccc}
\hline & \multicolumn{3}{c}{$\mathrm{N}^{\circ}$ Arboles/Clase diamétrica (cm.) } & \multicolumn{2}{c}{ Totales } \\
\cline { 2 - 7 } & $15-20(\mathrm{I})$ & $21-25(\mathrm{II})$ & $26-30(\mathrm{III})$ & $>30(\mathrm{IV})$ & Arb./clase & Latex (cc) \\
\cline { 2 - 7 } & 150 & 230 & 750 & 1000 & 4 & 2130 \\
& 175 & 300 & 450 & 850 & 4 & 1775 \\
& 60 & 100 & 675 & 1550 & 4 & 2385 \\
& 200 & 150 & & 450 & 3 & 800 \\
& 50 & 100 & & 825 & 3 & 975 \\
& & 375 & & & 1 & 375 \\
\hline S & 635 & 1255 & 1875 & 4675 & 19 & 8440 \\
Promedio & 127 & 209 & 625 & 935 & 2.7 & 444.21 \\
DS & 68.15 & 112.7 & 156.1 & 399 & & \\
\hline
\end{tabular}

seca, a partir del cual el volumen cosechado se va incrementando progresivamente hasta finales de lluvia en donde se obtiene la máxima producción. En las clases diamétricas mayores (26-30 y >30) se observa un pequeño descenso en los rango de producción en el 
periodo de plena lluvia que no afecta la tendencia creciente del volumen total cosechado, por el contrario la diferencia entre este periodo y el de fines de lluvia es mínima.

Respecto a la producción de látex promedio, registrada por cada clase diamétrica en los cuatro periodos de extracción, se ha determinado que es directamente proporcional al diámetro (Tabla 8). Los incrementos porcentuales en la producción de látex de una clase diamétrica inferior a la superior se observa que al inicio es creciente; en cambio de la tercera clase a la cuarta disminuye, $18 \%$ en el mes de mayo, $59 \%$ en septiembre, $33 \%$ en el mes de diciembre, mientras que en el marzo hay un incremento de $3 \%$. Esto es un indicador de que si bien a mayor diámetro mayor producción, también existe una disminución en el incremento porcentual de la producción a partir de un cierto diámetro, en este caso $30 \mathrm{~cm}$.

De otro lado los resultados de los Tablas 7 y 8 nos muestran que la producción promedio de látex de sangre grado es también directamente proporcional al aumento de las precipitaciones. Iniciando en periodo seco hacia inicio de lluvia con un incremento de $83 \%$, de inicio de lluvia a plena lluvia el incremento es de $10.5 \%$ y de plena lluvia a fines de lluvia el incremento solo alcanzó a $4.8 \%$; mientras que de fines de lluvia a la época seca la producción disminuyó en $53 \%$, esto nos señala una fuerte incidencia de la abundancia de lluvias en el almacenamiento de látex por la planta, a mayor precipitación mayor volumen de cosecha.

Según estos resultados se recomiendan no extraer el látex en épocas secas (junio a noviembre), pero si en los meses de diciembre a mayo donde las precipitaciones son más frecuentes y la producción se incrementa según los resultados obtenidos.

Relación diámetro / producción.

Para determinar la relación del diámetro con la producción de látex de
Tabla 9. Producción (cc) promedios de látex de sangre de grado en cuatro periodos de lluvias.

\begin{tabular}{lccccc} 
& \multicolumn{4}{c}{ Clase de diámetro (cm.) } & \multirow{2}{*}{$\mathrm{R}^{2}$} \\
\cline { 2 - 5 } Periodo de lluvias & $15-20$ & $21-25$ & $26-30$ & $>30$ & 0.9508 \\
\hline Fines (Mayo) & 171 & 291 & 682 & 1112.5 & 0.935 \\
Inicio (Diciembre) & 127 & 209 & 625 & 935 & 0.9337 \\
Plena lluvias (Marzo) & 115.6 & 369 & 550 & 865 & 0.9887 \\
Periodo seco (Septiembre) & 76 & 151 & 392 & 408 & 0.8929 \\
\hline Promedio & 122.4 & 255 & 562 & 830 &
\end{tabular}

realizó un análisis de regresión simple, con los promedios según clase diamétrica, en las poblaciones naturales de bosques secundarios de sangre de grado, determinándose una relación directa entre estas variables en los cuatro periodos de clima: Fines de lluvia $\left(\mathrm{R}^{2}=0.9508\right)$, época seca $\left(\mathrm{R}^{2}=0,8929\right)$, inicio de lluvia $\left(\mathrm{R}^{2}=0.9337\right)$ y plena lluvia (marzo) $\left(\mathrm{R}^{2}=\right.$ 0.9889); así mismo la producción de látex tiene una relación directa con el incremento del diámetro es decir a mayor diámetro mayor producción, como se puede ver en la Figura 1 y Tabla 9, siendo mayores las regresiones en los periodos de plena lluvia (marzo) y

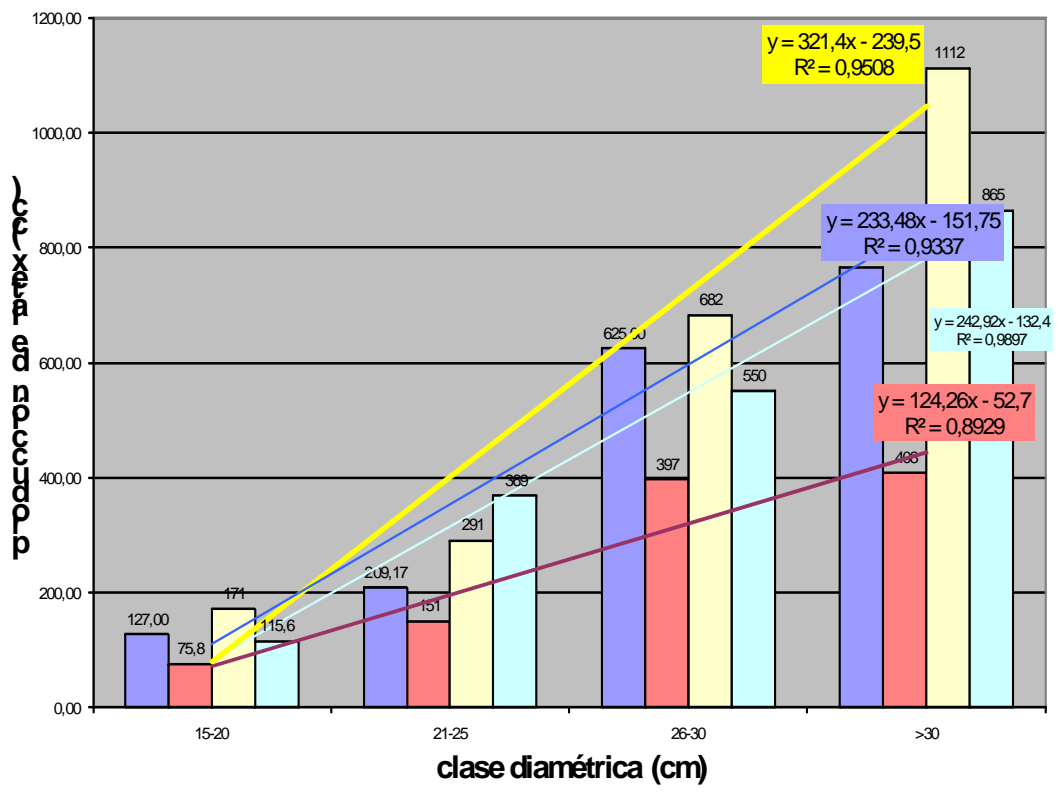

\begin{tabular}{clll}
\hline diciembre & $\square$ setiembre & $\square$ mayo & $\square$ marzo \\
Lineal (diciembre) & - Lineal (setiembre) & - Lineal (mayo) & - Lineal (marzo) \\
\hline
\end{tabular}

Figura 1. Producción de látex de Croton sp. por periodo en la localidad de Padre Abad, Ucayali Perú (2001).

fines de lluvia (mayo).

Tabla 8. Producción (cc) e incremento promedio de látex por clase diamétrica y periodos de lluvias.

\begin{tabular}{|c|c|c|c|c|c|c|c|c|}
\hline \multirow{2}{*}{$\begin{array}{l}\text { Clase diamétrica } \\
(\mathrm{cm} .)\end{array}$} & \multicolumn{8}{|c|}{ Producción (cc) / Periodos de pp. } \\
\hline & Мayo & Inc. $\%$ & Set. & Inc. \% & Dic. & Inc. \% & Marz. & Inc. $\%$ \\
\hline $15-20$ & 171 & & 75.88 & & 127 & & 115.6 & \\
\hline $21-25$ & 291 & 41 & 151.67 & 50 & 209 & 39 & 369 & 69 \\
\hline $26-30$ & 682 & 57 & 397.50 & 62 & 625 & 66 & 550 & 33 \\
\hline$>30$ & 1112.50 & 39 & 408.33 & 3 & 935 & 33 & 865 & 36 \\
\hline
\end{tabular}

sangre de grado y los niveles de precipitación se 


\section{Conclusiones.}

- Se determinó que existe alta variabilidad de producción de látex por árbol, dentro y entre las clases diamétricas estudiadas.

- Se determinó que la producción de látex de sangre de grado es directamente proporcional al DAP y al incremento de las precipitaciones.

- La mayor producción de látex se da en el periodo de fines de lluvia (mayo) y en los diámetros mayores a $30 \mathrm{~cm}$ de DAP, siendo la tendencia igual en los tres periodos de precipitación estudiados.

- El volumen de producción en litros de sangre de grado por periodos de extracción, es variable: disminuyó en $53 \%$ de fines de lluvia al periodo seco, se recuperó la producción en $83 \%$ al inicio de lluvias, $10.5 \%$ de inicio de lluvia a plena lluvia y $4.8 \%$ de plena lluvia a fines de lluvia.

\section{Agradecimientos.}

Debemos nuestro reconocimiento a los siguientes agricultores que voluntariamente han colaborado aportando los árboles y participado directamente en el estudio de extracción del látex de sangre de grado, señores: Moisés Quinto Quispe, Nery Salas Fatama, Lirio Rojas Isidro, Antonio Rueda Pantoja, y Feliciano Santa María Álvarez y Alberto Condezo Álvarez; así como a sus familiares por las facilidades brindadas. A Wilson Saldaña M. y Edvin Quito Ponce personal de apoyo del IIAP Ucayali.

\section{Literatura citada.}

Atanasio N. \& Domínguez G. 2000. Evaluación preliminar de la producción de látex de Croton draconoides $\mathrm{M}$. Arg. En bosques secundarios de la provincia de Padre Abad, Ucayali. Revista Forestal del Perú. Vol. XXIII, $\mathrm{N}^{\circ} 1$ y 2. Pag. 39-50.

Forero E. 1992. Etnobotanical observatións en Croton lechleri, Muell Arg. In the Amazon Valley (Colombia). Final reportsubmitted to shaman Pharmaceuticals Inc. Colombia.

Gaviria A. 1995. Bases para el aprovechamiento de sangre de grado en la selva Central. Proyecto especial Pichis - Palcazú.

Gudiño E., Gutiérrez F. \& Espinosa S. 1991. Lineamientos preliminares para el manejo de Croton spp. en la Amazonía Ecuatoriana. Shaman Pharmaceuticals. Inc. Propietary informatión. Quito, Ecuador.

INIAA-JICA - Instituto Nacional de Investigación Agraria y Agroindustrial. 1999. Estudio Conjunto sobre Investigación y Experimentación en Regeneración de
Bosques en la Zona Amazónica de la República del Perú. Informe Final de la Agencia de Cooperación Internacional del Japón-JICA. (III).

King R., Meza E., Ayala L., Forero E., Peña M., Zak V. \& Bastien H. 1997. Croton lechleri \& the sustainable harvest \& management of plants in pharmaceuticals, phymedicines and cosmetics industries. In international symposium en Herbal medicine, warkshop IIIEnvironmental Protection Censers. Honoluluu, Hawai: 8-10.

Lao M.R. \& Flores S. 1972. Árboles del Perú. Descripción de algunas especies forestales del Genaro Herrera - Universidad Nacional Agraria La Molina (UNALM). Lima - Perú. Cooperación Técnica del Gobierno Suizo.

Meza E. 1998. El Manejo Sostenible de Sangre de Drago o grado Material Educativo. Shaman Pharmaceticals, INC.

Meza E. 1999. "Sangre de Grado” y el reto de su producción sustentable en el Perú. Universidad Nacional Mayor de San Marcos.

Nalvarte A., De Jong W. \& Domínguez G. 1999. Plantas amazónicas de uso comercial: Diagnostico de un sector económico con un potencial de realización. Lima, Perú. CIFOR/UNALM.

Pinedo M., Rengifo E. \& Cerruti T. 1997. Plantas medicinales de la Amazonia Peruana. Estudio de uso y Cultivo. TCA. Lima, Perú.

Phillips O. 1991. La sangre de grado en el Dpto. de Madre de Dios situado en el sur Occidental del Perú. Shaman Pharmaceuticals, Inc, Propietary information, Lima, Perú.

Ravelo N. 1992. Regeneración natural de sangre de grado, Croton spp., en el alto Napo, Ecuador. Alarcón, Soldi \&MENA (Eds). Etnobotánica y Valoración de los Recursos Florísticos Silvestres en el Alto Napo, Ecuador. Universidad Técnica del Norte, Ibarra, Ecuador: $129-155$.

Ravelo N. 1994. Valor Económico, Usos y Métodos de Extracción de Látex de sangre de grado, Croton spp., en el Alto Napo, Ecuador. Alarcón, Soldi \&Mena (Eds). Etnobotánica y Valoración de los Recursos Florísticos Silvestres en el Alto Napo, Ecuador. Universidad Técnica del Norte, Ibarra, Ecuador: 155 - 173.

Ríos O. 2006. Distribución natural y determinación edafoclimatica de Croton lechleri Muell.Arg. (Sangre de grado) en bosques secundarios de Ucayali. Universidad Nacional de Ucayali. Facultad de Ciencias Forestales. Tesis para optar el título de Ing. Forestal. Pucallpa-Perú.

Chen Zp. 1994. Studies on the anti-tumour, anti bacterial and wound-healing propierties of Dragon's blood. Consultado diciembre del 2009. Disponible en http:/www.mdidea.com/products/proper/proper027resear ch.html.

\footnotetext{
${ }^{1}$ Anterior: Instituto de Investigación de la Amazonia Peruana, Pucallpa, Perú. Actual: Universidad Nacional Intercultural de la Amazonia Peruana - Yarinacocha. Pucallpa, Ucayali Perú. acastillo_2519@hotmail.com

${ }^{2}$ Universidad Nacional Agraria La Molina, Av La Universidad s/n-La Molina, Lima Perú. gdominguez@lamolina.edu.pe
} 